\title{
Misdiagnosis of osteosarcoma as cementoblastoma from an atypical mandibular swelling: A case report
}

\author{
ZAO FANG $^{1 *}$, SHUFANG JIN ${ }^{1 *}$, CHENPING ZHANG ${ }^{1}$, LIZHEN WANG $^{2}$ and YUE HE ${ }^{1}$ \\ ${ }^{1}$ Department of Oral Maxillofacial Head and Neck Oncology, Faculty of Oral and Maxillofacial Surgery; \\ ${ }^{2}$ Department of Oral Pathology, Shanghai Ninth People's Hospital, Shanghai Jiao Tong University School of Medicine, \\ Shanghai Key Laboratory of Stomatology, Shanghai 200011, P.R. China
}

Received December 1, 2014; Accepted January 12, 2016

DOI: $10.3892 / 01.2016 .4433$

\begin{abstract}
Cementoblastoma is a form of benign odontogenic tumor, with the preferred treatment consisting of tooth extraction and follow-up examinations, while in certain cases, follow-up examinations without surgery are performed. Osteosarcoma of the jaw is a rare, malignant, mesenchymal tumor, associated with a high mortality rate and low incidence of metastasis. Cementoblastoma and osteosarcoma of the jaw are dissimilar in terms of their histological type and prognosis; however, there are a number of covert associations between them. The present study describes the case of a 20-year-old female with an unusual swelling in the left mandible that had been present for 2 years. The patient was initially clinically diagnosed with cementoblastoma; however, following pathological examination, a diagnosis of osteosarcoma was confirmed. A total mandibulectomy was performed on the left mandible, with a fibular osteomyocutaneous flap used for reconstruction. At the 6-month post-operative examination, the patient demonstrated normal occlusion and oral commissure, and no recurrence or metastasis was observed over 2 years of follow-up.
\end{abstract}

\section{Introduction}

According to the World Health Organization, cementoblastoma is classified as a benign odontogenic tumor (1). The majority of patients exhibit no marked clinical signs, with the tumors often being identified following orthopantomography. Typical treatment of cementoblastoma includes the complete removal

Correspondence to: Professor Yue He, Department of Oral Maxillofacial Head and Neck Oncology, Faculty of Oral and Maxillofacial Surgery, Shanghai Ninth People's Hospital, Shanghai Jiao Tong University School of Medicine, Shanghai Key Laboratory of Stomatology, 639 Zhi Zao Ju Road, Shanghai 200011, P.R. China E-mail: william5218@126.com

*Contributed equally

Key words: cementoblastoma, osteosarcoma, oral and maxillofacial surgery, histopathology, radiology of the lesion with extraction of the associated tooth (2); however, certain patients may decide against surgery, undergoing follow-up alone. Osteosarcoma is a non-hematopoietic, malignant tumor of the bone, with the neoplastic cells of the lesion producing osteoid (3). This form of tumor is characterized by high malignancy, metastasis and mortality rates (4). The tumors are most prevalently located in the metaphyseal region of long bones, particularly in the knee and pelvis (5). Osteosarcoma of the jaw is rare, accounting for 5-13\% of all osteosarcoma cases (6), the majority of which are located in the mandible. Until recently, the etiology of osteosarcoma of the jaw has remained obscure. In a series of patients, a greater number of differences in gender, tumor subtype and metastatic potential of osteosarcoma of the jaw were noted than previously observed, and better imaging, earlier diagnosis and more aggressive treatment has been indicated to improve the surgical clearance of the tumor (7). The current study reports the case of a young female presenting with an unusual swelling in the left mandible.

\section{Case report}

In December 2012, a 20-year-old female presented with a 2-year history of swelling in the left mandible, without pain or any discomfort. The only accompanying symptom was tooth mobility of the molars. At 1 month prior to presentation, the swelling had progressed at an increased rate, and was associated with significant pain and numbness. The patient was initially examined by a dentist who performed an orthopantomography examination. The resulting images exhibited a well-defined, high-density change of the alveolar ridge surrounding the first left mandibular molar, with a peripheral radiolucent band. Following orthopantomography (ORTHOPHOS XG 5 DS/Ceph; Sirona Dental Systems GmbH, Bensheim, Germany), the initial clinical diagnosis was stated as cementoblastoma (Fig. 1). As the leading treatment for cementoblastoma, extraction of the first left mandibular molar was performed. Subsequent to the diagnosis and molar removal, the patient was transferred to the Department of Oral Maxillofacial Head and Neck Oncology, Ninth People's Hospital (Shanghai, China), for further treatment. A physical examination identified that the lesion was a $2.5 \times 2.5-\mathrm{cm}$ solid swelling on the left mandible, with the primary expansion observed on the buccal 


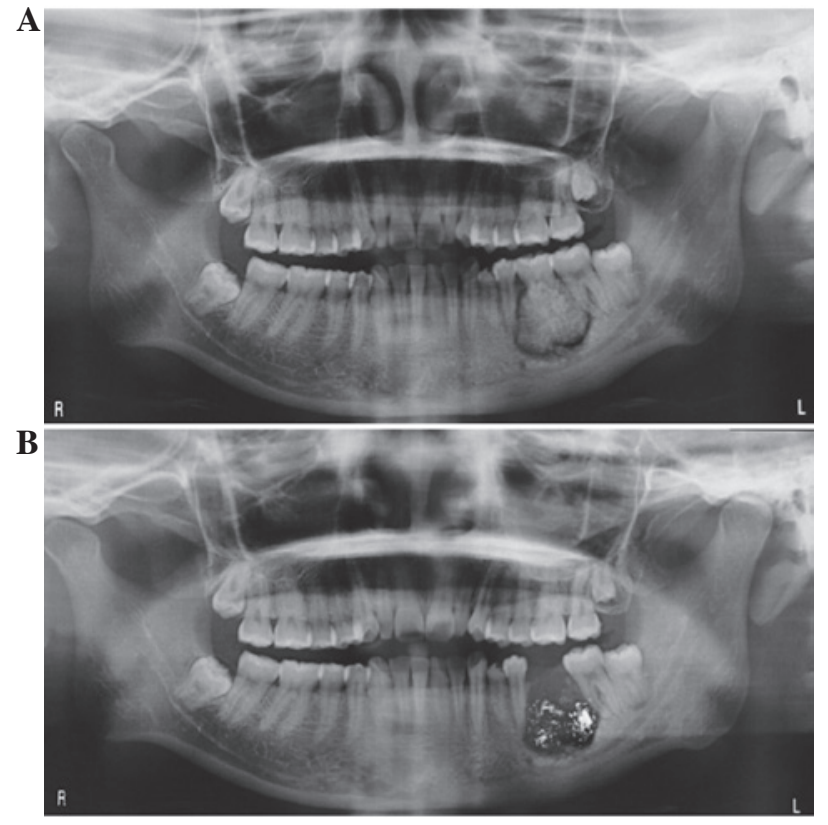

Figure 1. Pre-operative orthopantomography images (A) prior to and (B) following initial tooth extraction.

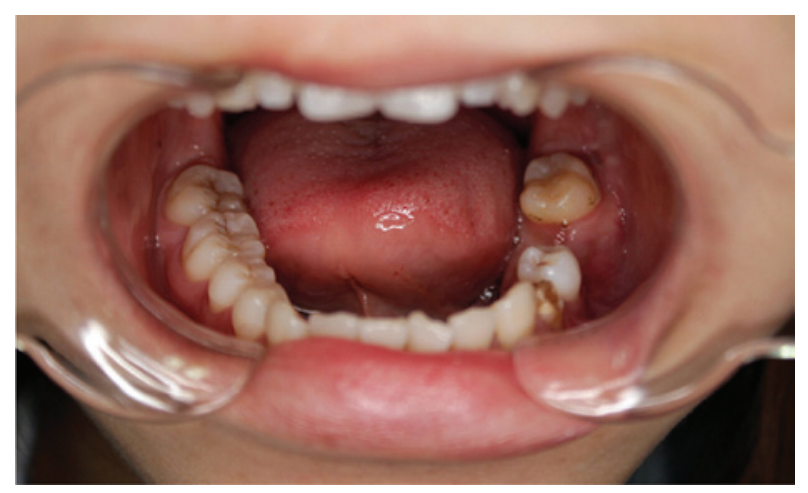

Figure 2. Pre-operative intraoral view of the swelling in the left mandible.

cortical plate (Fig. 2). No enlarged lymph node was noted in the cervicofacial chain. Further orthopantomography was performed, and detected that the region of radiolucency, with a rough margin, involved the roots of the second premolar and the second molar. Certain high-density spots were identified in the radiolucent area (Fig. 1). A chest radiograph appeared to reveal no suspicious abnormalities. The patient underwent a left total mandibulectomy with reconstruction using a vascularized fibular osteocutaneous flap and titanium plate (Fig. 3). The tumor exhibited typical characteristics of osteosarcoma, including the compact bone and trabecula-like structures surrounded by abundant active osteoblasts, with the final pathology confirming the lesion to be sclerosing osteosarcoma (Fig. 4). No radiotherapy or chemotherapy was administered to accompany the radical surgical. The patient recovered well and was discharged 14 days after surgery without any other complications. At a 6-month follow-up examination, the patient exhibited normal occlusion and oral commissure (Figs. 5 and 6), and no recurrence or metastasis was observed during 2 years of follow-up, using enhanced CT scans of the head, neck and chest regions.
A

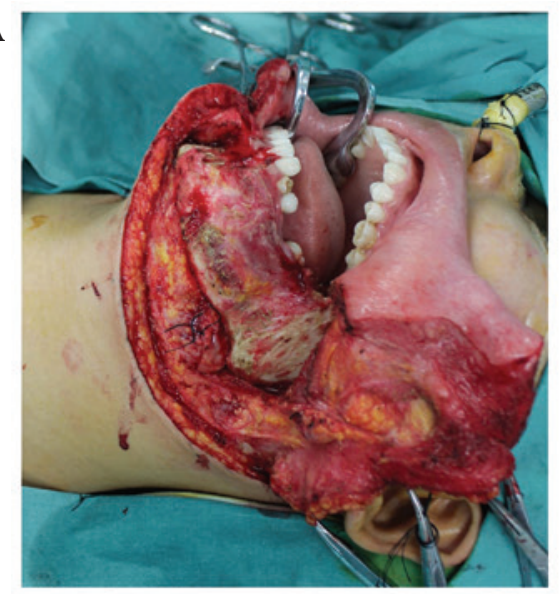

B

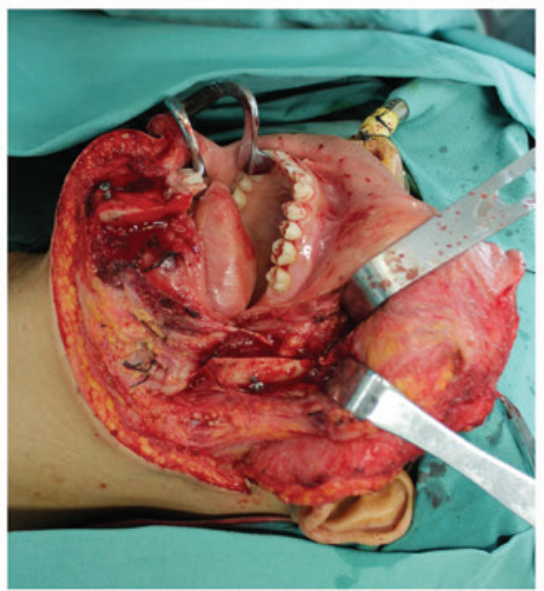

C

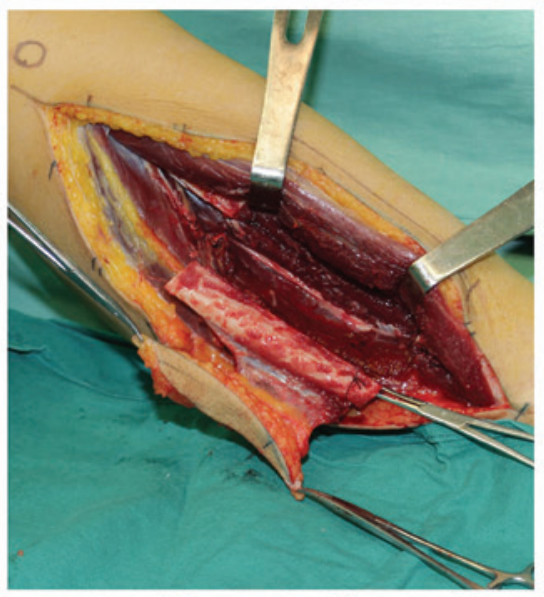

D

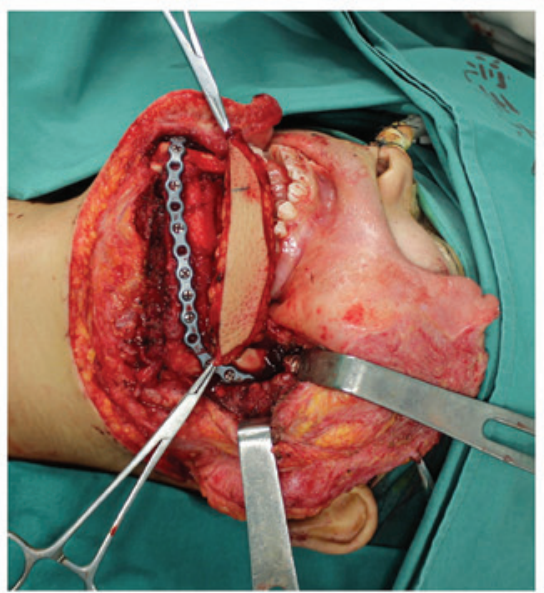

Figure 3. Intraoperative images. (A) The mass with exposure of the mandible; (B) the rest of the mandible; (C) the vascularized fibula flap; and (D) the fibula flap used to reconstruct the mandible and mucosa. 


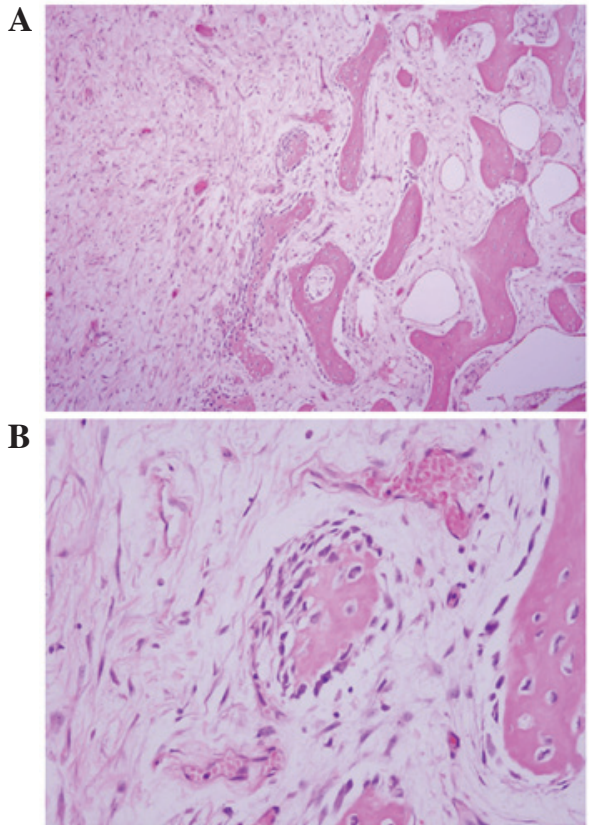

Figure 4. Histopathological analysis of the center section of the tumor tissue exhibiting irregular osteoid surrounded by osteoblasts, which have abnormal mitoses, at a magnification of (A) x200 and (B) x400. Staining, hematoxylin and eosin (Baso Diagnostics, Inc., Zhuhai, China).

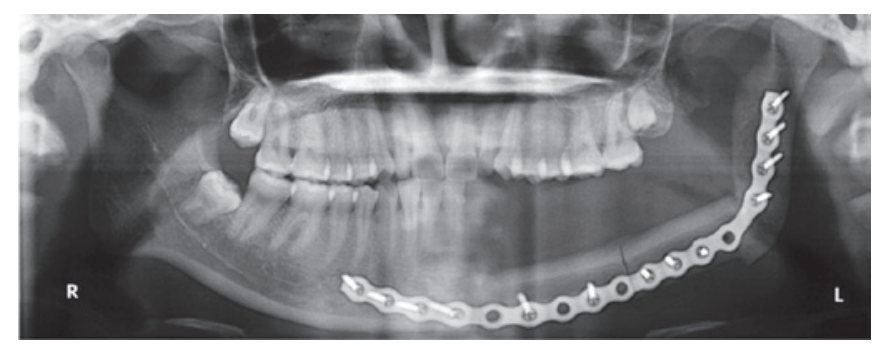

Figure 5. Post-operative orthopantomography showing the mandible reconstructed with a free fibula osteomyocutaneous flap and titanium plate.

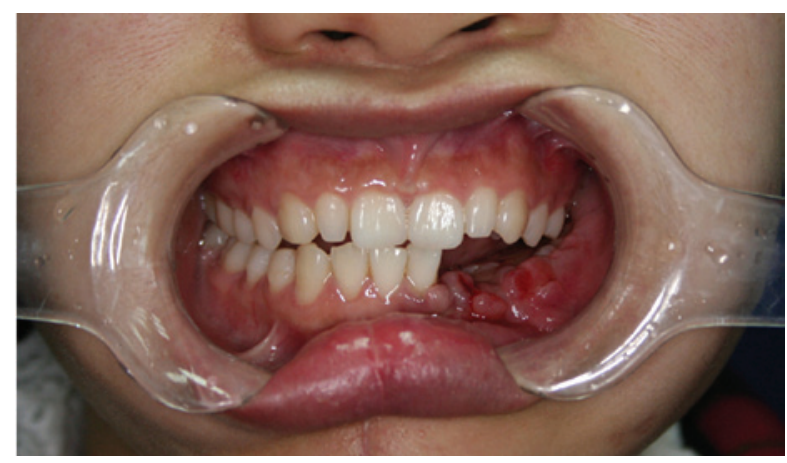

Figure 6. Post-operative occlusion and appearance similar to that observed pre-operatively.

\section{Discussion}

True cementoma, now known as cementoblastoma, is a benign odontogenic tumor commonly presenting with painful swelling of the alveolar ridges (1). Such lesions have a predilection for the mandible and are primarily associated with the mandibular first molar. The pulp vitality of the involved teeth generally remains intact. Cementoblastoma is most commonly observed in males at a mean age of 21 years (8). The majority of patients are usually entirely asymptomatic, and diagnosis is often made according to X-ray observations. Certain patients experience swelling, pain, tooth mobility, paresthesia or cortical expansion of the mandible. In the current case, the only initial clinical sign of the disease was swelling. The imaging data obtained from the patient's dentist appeared to be similar to the radiographical appearance of cementoblastoma, which includes the formation of hard-tissue in connection with the root of a tooth, a definite boundary, a radiopaque or mixed-density and a thin surrounding radiolucent zone (1). In the present case, 1 month prior to presentation, the mandibular began to swell at an increased rate, the pain was also increasing and numbness was experienced. An important detail was later noted to have been overlooked in the initial orthopantomography; the roots of the involved tooth had been eroded by the tumor and presented an incomplete structure of the teeth. When combined with the patient symptoms, this finding meant that concern was raised over the diagnosis of cementoblastoma.

Osteosarcoma is a malignancy of mesenchymal cells and commonly occurs in the long bones. Osteosarcoma of the jaw is rare, accounting for $\sim 1 \%$ of all head and neck malignancies (9). The disease has a moderate male predilection, with a peak in incidence in the second to third decades of life (10). The maxilla and the mandible are affected almost equally, with the alveolar ridge and the antrum in the maxilla and the body of the mandible serving as preferential sites for the tumors. A number of risk factors are understood to be associated with osteosarcoma, including genetic bias, Paget's disease of the bone, hereditary retinoblastoma, trauma, a history of fibrous dysplasia, chromic oxide and radiation (11). However, the disease etiology remains unclear.

Clinical symptoms of osteosarcoma of the jaw include swelling (with or without pain) and loosening of the teeth. Certain well-differentiated tumors grow slowly and present as benign lesions. As a malignant tumor, osteosarcoma is invasive, and may cause irreversible numbness if the neoplasm invades the inferior alveolar nerve (10). This form of numbness is dissimilar to the paresthesia experienced by patients with cementoblastoma, which is caused by tumor pressure. Occasionally, laboratory parameters have demonstrated an increase in alkaline phosphatase or lactic dehydrogenase in serum levels (12), with such unfavorable serum parameters considered to indicate a poor prognosis for patients with osteosarcoma.

In general, the radiographical appearance of osteosarcoma of the jaw relies on three processes: i) Bone formation and mineralization; ii) bone destruction; and iii) periosteal bone formation. In the majority of cases, the tumor is a mixed lytic/blastic lesion that is dependent on the degree of ossification (13). During the early stage of osteosarcoma, the observation of a widened periodontal ligament space and inferior dental canal is typical of the disease, however, it is extremely difficult to recognize (14). In certain cases, the typical 'sunray' appearance may be observed at the periphery of the tumor in plain radiographs and computed tomography scans. The hallmark of cementoblastoma is the sharp border between the tubular dentin of the root and the cementum-like 
component (15). In the present case, the osteosarcoma resembling cementoblastoma mimicked 'benign' bone growth, and there was no observation of the typical 'sunray' radiographical appearance. It was noted, however, that the tumor exhibited an irregular shape with a rough margin. The roots of the first molar and the mandibular canal were invaded by the tumor. The structure of the root canal was destroyed, and the wall of the mandibular canal was discontinuous.

Macroscopically, certain osteoblastic osteosarcomas tend to be grey-tan and randomly granular, while others become denser, sclerotic and more yellow-white (16). Histopathologically, the observation of osteoid is key for the diagnosis of osteosarcoma. Osteoid is a pink, dense, intercellular material that is curvilinear with small nubs and arborization (Fig. 4) (16). Sclerosing osteosarcoma is a subtype of osteoblastic osteosarcoma that presents with compact osteoid and bone within the tumor; certain studies have reported that the deposition of bone matrix occurs between adipocytes in the marrow cavity (16). As lesions are typically decalcified, the immature bone surrounded by adipocytes may be obscured (17). In certain cases, the osseous matrix may be deposited on the normal bone trabeculae, and the tumor cells may be small, pyknotic and surrounded by large amounts of bone matrix; this phenomenon is known as 'normalization' (16). When compared with the pathological characteristics of osteosarcoma, cementoblastoma is composed of sheets of cementum and peripheral radiating columns of unmineralized tissue, similar to normalization (18). For this reason, it is difficult to distinguish between cementoblastoma and osteosarcoma when only a small tissue specimen is available. A further feature of osteosarcoma is the variable amounts of cartilage matrix and fibrous tissue. In the present case, the tumor exhibited typical characteristics of osteosarcoma; it was composed of compact bone, trabecula-like structures, which were surrounded by abundant active osteoblasts with varying morphology and atypical nuclear fission, fibrous connective tissue and an infective lesion.

If the diagnosis of the current case were cementoblastoma, the optimal treatment would have been dental extraction and post-operative follow-up. Certain patients that present with no clinical signs may not decide to undergo surgery and instead undergo follow-up examinations alone. However, if a patient with osteosarcoma misdiagnosed as cementoblastoma underwent a dental extraction, the tumor would undergo rapid growth (19).

Without aggressive intervention, osteosarcoma of the jaw may be fatal, and prompt radical resection is currently the preferred treatment. Extensive resection requires surgeons to remove the tumor with a $1.5-2 \mathrm{~cm}$ margin. It has previously been reported that the 5-year survival rate of patients with osteosarcoma of the mandible is $34.8 \%$ and that of the maxilla is $25.8 \%$ (20). Age serves as a prognostic factor of osteosarcoma, with older patients demonstrating a more favorable prognosis when compared with younger patients (21). Local recurrence and metastasis may also present in patients. Metastasis generally occurs via the bloodstream, and most frequently arises in the lungs (22). Regional lymph node metastasis is rarely observed. Metastasis occurs in only $18 \%$ of patients with osteosarcoma of the jaw, and it has been reported that men are affected more commonly (21). Using an immunomagnetic detection assay, Bruland et al (23) examined blood and bone marrow samples from 60 patients who were suspected of having osteosarcoma. It was demonstrated that this type of technique may be successfully used to detect metastasis in cases of osteosarcoma. In the present case, the chest radiograph did not detect any metastases. At a 2-year follow-up examination, the patient demonstrated no signs of local recurrence or metastasis.

A large amount of controversy surrounds the performance of post-operative radiotherapy and chemotherapy. Due to the anatomical circumstances in the craniofacial region, it is challenging to achieve a tumor-free surgical resection margin. The majority of scholars consider that patient survival time is limited if the osteosarcoma is treated by ablative surgery alone. However, other studies have suggested that extensive resection alone is sufficient, with the 5-year survival rate of patients who underwent resection recorded at $\sim 60 \%(24,25)$. It is considered that radiotherapy should be confined to the treatment of unresectable, residual and recurrent tumors, as radiotherapy itself is a risk factor for osteosarcoma. From the results of previous studies, it cannot be confirmed whether chemotherapy has an effect on the prognosis of patients with osteosarcoma $(26,27)$; this may be due to the diversity in chemotherapy regimens that were administered. A number of previous studies reported that modern adjuvant chemotherapy increased the survival time of patients, and in certain cases, also controlled metastasis (10).

Cementoblastoma is a type of benign odontogenic tumor, with the optimal treatment consisting of tooth extraction and follow-up examination. Osteosarcoma of the jaw is a rare, malignant disease with a poor prognosis, and the imaging and clinical appearance of the lesion is highly variable. The preferred treatment options include a segmental mandibulectomy or maxillectomy, whilst post-operative radiotherapy and chemotherapy is controversial. Due to the opposing biological behavior underlying the two diseases and varying prognoses, it is important that surgeons are able to distinguish between osteosarcoma and cementoblastoma. The current case presented with sclerosing osteosarcoma, which originated from the mandible, and resembled a cementoblastoma. The findings discussed in the present study aim to provide a clearer understanding of the distinguishing features of osteosarcoma and cementoblastoma, and possibly aid their differentiation in the clinic.

\section{Acknowledgements}

This study was supported by grants from the National Natural Science Foundation of China (grant no. 81271112), the Development Foundation supported by the Shanghai Municipal Human Resources and Social Security Bureau (grant no. 201312), and the Southern Management Corporation Rising Star Scholar, supported by Shanghai Jiao Tong University (grant no. 2013SMC-A-4).

\section{References}

1. van der Waal I: Cementoblastoma. In: World Health Organization Classification of Tumours. Pathology and Genetics of Head and Neck Tumours. Barnes L, Eveson JW, Reichart P and Sidransky D (eds). IARC Press, Lyon, p318, 2005.

2. Sharma N: Benign cementoblastoma: A rare case report with review of literature. Contemp Clin Dent 5: 92-94, 2014. 
3. Amaral MB, Buchholz I, Freire-Maia B, Reher P, de Souza PE, Marigo Hde A, Martins CR and Horta MC: Advanced osteosarcoma of the maxilla: A case report. Med Oral Patol Oral Cir Bucal 13: E492-E495, 2008.

4. Nthumba PM: Osteosarcoma of the jaws: A review of literature and a case report on synchronous multicentric osteosarcomas. World J Surg Oncol 10: 240, 2012.

5. Dahlin DC and Coventry MB: Osteogenic sarcoma. A study of six hundred cases. J Bone Joint Surg Am 49: 101-110, 1967.

6. Fu HH, Zhuang QW, He J, Wang LZ and He Y: Giant cell-rich osteosarcoma or giant cell reparative granuloma of the mandible? J Craniofac Surg 22: 1136-1139, 2011.

7. Granowski-LeCornu M, Chuang SK, Kaban LB and August M: Osteosarcoma of the jaws: Factors influencing prognosis. J Oral Maxillofac Surg 69: 2368-2375, 2011.

8. Brannon RB, Fowler CB, Carpenter WM and Corio RL: Cementoblastoma: An innocuous neoplasm? A clinicopathologic study of 44 cases and review of the literature with special emphasis on recurrence. Oral Surg Oral Med Oral Pathol Oral Radiol Endod 93: 311-320, 2002.

9. Ferrari D, Codecà C, Battisti N, Broggio F, Crepaldi F, Violati M, Bertuzzi C, Dottorini L, Caldiera S, Luciani A, et al: Multimodality treatment of osteosarcoma of the jaw: A single institution experience. Med Oncol 31: 171, 2014

10. Kämmerer PW, Shabazfar N, Vorkhshori Makoie N, Moergel M and Al-Nawas B: Clinical, therapeutic and prognostic features of osteosarcoma of the jaws - experience of 36 cases. J Craniomaxillofac Surg 40: 541-548, 2012.

11. Mendenhall WM, Fernandes R, Werning JW, Vaysberg M, Malyapa RS and Mendenhall NP: Head and neck osteosarcoma Am J Otolaryngol 32: 597-600, 2011.

12. Roca AN, Smith JL Jr and Jing BS: Osteosarcoma and parosteal osteogenic sarcoma of the maxilla and mandible: Study of 20 cases. Am J Clin Pathol 54: 625-636, 1970.

13. Cavalcanti MG, Ruprecht A and Yang J: Radiological findings in an unusual osteosarcoma in the maxilla. Dentomaxillofac Radiol 29: 180-184, 2000.

14. Gardner DG and Mills DM: The widened periodontal ligament of osteosarcoma of the jaws. Oral Surg Oral Med Oral Pathol 41: 652-656, 1976
15. Wang S, Shi H and Yu Q: Osteosarcoma of the jaws: Demographic and CT imaging features. Dentomaxillofac Radiol 41: 37-42, 2012.

16. Raymond AK, Ayala AG and Knuutila S: Conventional osteosarcoma. In: World Health Organization Classification of Tumours. Pathology and Genetics of Tumours of Soft Tissue and Bone. Fletcher CDM, Unni KK and Mertens F (eds). IARC Press, Lyon, pp264-270, 2002.

17. Klein MJ and Siegal GP: Osteosarcoma: Anatomic and histologic variants. Am J Clin Pathol 125: 555-581, 2006.

18. Jaffe HL: Intracortical osteogenic sarcoma. Bull Hosp Joint Dis 21: 189-197, 1960.

19. Nissanka EH, Amaratunge EA and Tilakaratne WM Clinicopathological analysis of osteosarcoma of jaw bones. Oral Dis 13: 82-87, 2007

20. Patel SG, Meyers P, Huvos AG, Wolden S, Singh B, Shaha AR, Boyle JO, Pfister D, Shah JP and Kraus DH: Improved outcomes in patients with osteogenic sarcoma of the head and neck. Cancer 95: 1495-1503, 2002.

21. Garrington GE, Scofield HH, Cornyn J and Hooker SP: Osteosarcoma of the jaws. Analysis of 56 cases. Cancer 20: 377-391, 1967.

22. Anil S, Krishnan AP and Rajendran R: Osteosarcoma of the mandible masquerading as a dental abscess: Report of a case. Case Rep Dent 2012: 635062, 2012.

23. Bruland OS, Høifødt H, Saeter G, Smeland S and Fodstad O: Hematogenous micrometastases in osteosarcoma patients. Clin Cancer Res 11: 4666-4673, 2005.

24. Nagler RM, Malkin L, Ben-Arieh Y and Laufer D: Sarcoma of the maxillofacial region: Follow-up of 25 cases. Anticancer Res 20: 3735-3741, 2000

25. Yamaguchi S, Nagasawa H, Suzuki T, Fujii E, Iwaki H, Takagi M and Amagasa T: Sarcomas of the oral and maxillofacial region: A review of 32 cases in 25 years. Clin Oral Investig 8: 52-55, 2004.

26. Jundt $\mathrm{G}$ and Prein J: Bone tumors and tumor-like lesions of the jaw. Findings from the Basel DOSAK reference registry. Mund Kiefer Gesichtschir 4 (Suppl 1): S196-S207, 2000 (In German).

27. Mardinger O, Givol N, Talmi YP and Taicher S: Osteosarcoma of the jaw. The chaim sheba medical center experience. Oral Surg Oral Med Oral Pathol Oral Radiol Endod 91: 445-451, 2001. 